\title{
Maze running in kangaroo rats with sensory deprivations
}

\author{
MOLLY WEBSTER and DOUGLAS B. WEBSTER \\ Kresge Hearing Research Laboratory of the South, Louisiana State University Medical Center, New Orleans, Louisiana 70119
}

\begin{abstract}
Kangaroo rats trained to a complex maze were run in it with and without sensory deprivations; data were collected for time, errors, collisions, and percent of trials completed. Only blinding had a significant effect on performance by itself; cutting the vibrissae in combination with blinding had a secondarily strong effect. Deafening (but not olfactory bulbectomy) combined with blinding and cutting the vibrissae also had a strong effect. Bulbectomy had no effect except when combined with blinding, deafening, and cutting the vibrissae. Maze running may be related to activity within the burrow and to surface activity at night, and these activities are discussed in light of these results.
\end{abstract}

Kangaroo rats spend the day in underground burrows which are sometimes quite complex, and they spend much of the night in aboveground activities which include foraging. Their presence in an area can often be detected by paths or runways worn through the grass. In short, an activity similar to maze running seems to be part of their natural repertoire, and it is done under conditions of partial sensory deprivation (e.g., in near total darkness).

This experiment was designed to test the sensory cues important for this type of activity. It was particularly prompted by other work we have done which indicated that while either audition or vision can be used for predator avoidance, the loss of both is a serious handicap (Webster \& Webster, 1971). Therefore, in the present experiment, we were interested not in acquisition of a maze habit, but in the ability of the kangaroo rat to run a previously learned maze without one or more senses; and in the performance deficits caused by different states of sensory deprivation.

\section{METHOD}

\section{Subjects}

In all, 53 wild-caught kangaroo rats (Dipodomys merriami) of unknown ages were used. Data were obtained for 31 . The others did not achieve criterion in a reasonable time and were discontinued, or were intractable in this situation (e.g., they continually leaped out of the maze), or, in a few cases, died of injury or unknown causes. Deaths were apparently unrelated to treatments. All were weighed daily; while weight loss was fairly common immediately after surgery, most animals from whom data were obtained maintained approximately the same weight during a "block" of trials-that is, after any surgery until the next.

Between training and testing sessions, the subjects were housed in individual plastic cages in a quiet room and provided with rolled oats ad lib, and occasionally with lettuce. Kangaroo

This work was supported by U.S.P.H.S. Grant NS-11459 from NINDS to Douglas B. Webster. The authors express their appreciation to $J$. W. Klara and $M$. J. Webster for assistance in data collection, to Dr. C. I. Berlin for ultrasonic monitoring, and to Drs. L. F. Hughes and J. K. Cullen, Jr. for help and advice on statistical analyses; and particularly to Dr. Hughes for a critical reading of the manuscript. rats normally do not drink, and it is unnecessary to provide them with water.

\section{Apparatus}

The animals were trained and tested for 10 trials a day in a maze constructed of black lucite with sides $12.5 \mathrm{~cm}$ high and alleys $10 \mathrm{~cm}$ wide; it had nine possible errors (seven of which were blind alleys) and eight turns necessary to reach the goalbox, arranged in the following order: R, R, R, L, R, L, L, L.

The maze was placed in a sound-shielded IAC chamber to minimize extraneous sensory information, but the test situation itself richly abounded in sensory cues. The size of the maze permitted tactile input: the sides of the passages are taller than a running kangaroo rat but not as tall as a kangaroo rat in its bipedal stance; the alleys are narrower than the tip-to-tip length of the animals' vibrissae. The maze was illuminated by a $60-W$ bulb, either suspended above the maze or placed directly below it. The maze was swept when sand or debris accumulated, but in order to preserve olfactory cues, it was not washed.

\section{Procedure}

The animals were run in groups of three, at different times and in a different order within their group each day. Two identical numbered boxes for each animal contained sand or kitty litter and a bit of food; an animal was released into the maze from one box (via a guillotine door) and collected and held in the other box at the end of the maze. It remained in this second box while the other two animals in the group were running and, thus, usually had an intertrial interval of 2-3 min. Before the first trial of each session, and before other trials as necessary, a drop of oil was placed on the animal's back, between the shoulders; for most kangaroo rats, this serves as a motivation to run a maze and sand bathe in the sand in the goalbox.

The maze was considered learned (criterion performance) when the animals had run 10 trials per day for 4 consecutive training days, with no interference from the experimenter, with at least 1 of the 4 days being error free, and with no significant variation in running times or errors over days as tested by ANOVA.

When the maze was learned, the animals were treated according to which of five experimental groups they had been randomly assigned to at the start of training. Those in four groups underwent sensory deprivations-ideally, one a week-in a different order for each group; those in the fifth group were simply anesthetized each week for 3 or 4 weeks.

All surgeries were done under chloral hydrate anesthesia $(.0076 \mathrm{cc} / \mathrm{g})$. A 2 -day recovery followed each surgery; then the animals were run for 4 days (40 trials) before undergoing the next deprivation (or anesthesia). Because some animals died 
before they had completed the series of four surgeries, it was occasionally necessary to do multiple surgeries in order to have enough individuals in each deprivation condition for statistical analyses.

Eyes were removed by mobilizing the ey eball, hemostating the vessels, cutting out the eyeball, and suturing closed the eyelid. Cochleae were destroyed by opening the middle ear through the tympanic membrane and crushing the cochlea with a pair of No. 5 watchmaker's forceps; a small piece of Gelfoam was packed into the wound, and the external auditory meatus was stitched closed. The olfactory bulbs were usually removed by aspiration through an opening in the frontal bones; occasionally they were destroyed by heat lesions. During both of these olfactory bulb surgeries, the entire cribriform plate was inspected before the wound was packed with Gelfoam and closed; thus, we were as confident as possible of having destroyed all the primary olfactory fibers. Vibrissae were merely clipped as close to the face as possible.

There were 16 experimental conditions: Criterion (CRIT: the 4 analyzed days before the first surgery); controls in their first, second, third, or fourth week past the criterion analy sis (N, NN, NNN, NNNN); and animals deprived of from one to four senses (C, O, E, V, CO, EV, ECO, VCO, EVO, EVC, EVCO; with C = cochleae, $\mathrm{O}=$ olfactory bulbs, $\mathrm{E}=$ eyes, $\mathrm{V}=$ vibrissae). All these groups contained five animals with the following exceptions: 31 in CRIT, 3 in NNNN, 7 in CO, 8 in EV, 9 in EVCO.

Data were collected for time, errors, and collisions for each of these 16 groups. A stopwatch was used to measure the time between a kangaroo rat's entrance in to the maze runway and its entrance into the goalbox. An error was counted when an animal entered a wrong turn or retraced its steps; a collision was counted when it collided with any wall of the maze. If the animal had not reached the goalbox in $5 \mathrm{~min}$, or if it remained motionless in the maze for $45 \mathrm{sec}$, it was removed from the maze, and the trial was considered incomplete. (This was the only type of interference that the experimenter performed once the maze was learned.) The analyses are based on the number of trials completed, rather than on the number started, since the latter method would indicate "no errors" for trials when the animal did not run at all.

\section{Postmortem Examinations}

After the experiment, most of the animals were anesthetized and perfused with saline followed by $10 \%$ formalin; the few animals that were found dead were simply immersed in $10 \%$ formalin. All heads were fixed in $10 \%$ formalin and then carefully dissected under a dissecting microscope and examined for the extent of the surgeries.

\section{RESULTS}

Comparisons among groups of control and sensory deprived animals were tested for significance using a modified Duncan's new multiple range test, in which the harmonic mean rather than the arithmetic mean was used in the calculation of the standard error (see Winer, 1962 for a discussion of the use of multiple range tests with unequal Ns). The level of significance was set as $\mathrm{p}<.01$ for all comparisons.

The means and standard errors of per-trial time, errors, and collisions were calculated for each of the 16 conditions. Since the animals in several conditions do not perform significantly differently, these conditions were grouped together, and the means and standard errors of these homogeneous subsets were calculated.
Figures 1-3 are graphs of the per-trial time, errors, and collisions for each condition or homogeneous subset. In all figures, the conditions are arranged in the same descending order of overall best performance, to facilitate comparisons. It can be seen that any given deprivation condition has a similar-if not identical-effect on all these dependent variables.

The effect of any condition on percent of trials completed was also similar to its effect on other dependent variables. All trials were completed in the CRIT and all $\mathrm{N}$ conditions, and in $\mathrm{C}, \mathrm{O}$, and $\mathrm{V}$ conditions; $94 \%$ were completed by $\mathrm{CO}$ animals and $99 \%$ by VCO. All conditions without eyes completed about $85 \%$ of trials, except that EVC completed only $62 \%$ and EVCO only $30 \%$.

The effect of destroying the chochleae (C) was seen only when it was combined with other serious losses; $\mathrm{C}$, $\mathrm{CO}$, and VCO animals performed like normals; ECO animals performed like $E$ animals on time, errors, and percent run, and both groups performed like normals on collisions. EVC animals performed significantly worse than all other groups except EVCO, showing the effect of $\mathrm{C}$ in combination with $\mathrm{E}$ and $\mathrm{V}$.

Similarly, ablation of the olfactory bulbs $(0)$ had but little effect except in the case of the EVCO group: $O$, $\mathrm{CO}$, and VCO animals performed like normals; ECO animals performed like Es, and EVO animals like EVs on time and errors, and all four groups were similar on percent of trials completed; on collisions, ECOs were like normals, but EVOs were significantly different from all other groups with olfactory bulb ablations.

Cutting the vibrissae (V) had no effect except in combination with $\mathrm{E}: \mathrm{V}$ and VCO animals were like normals on all dependent variables. EV and EVO animals were alike on time and significantly slower than $E$ and ECO animals, while EVC and EVCO animals were each significantly slower still. EV animals made more errors than E and ECO animals although not quite significantly more; however, EVO animals made significantly more errors than the other three and also formed a homogeneous subset with EV animals. Cutting the vibrissae in combination with $\mathrm{E}, \mathrm{EO}$, or $\mathrm{EC}$ had a significant effect on numbers of collisions, each group being worse than the last. EVCO animals made fewer collisions per trial than EVO animals, which probably reflected the very long per-trial times for the former group.

Removing the eyes (E) was the only procedure which, by itself, had an effect on the maze running of kangaroo rats. On both times and errors, all $\mathrm{E}$ groups were significantly worse than normals, and they completed fewer trials; on collisions, however, E did not have an effect except when combined with $\mathrm{V}$.

Animals with all four senses removed (EVCO) still ran the maze $30 \%$ of the time, although it took them 13 times longer than normals and half again as long as animals deprived of eyes alone; their errors were about 


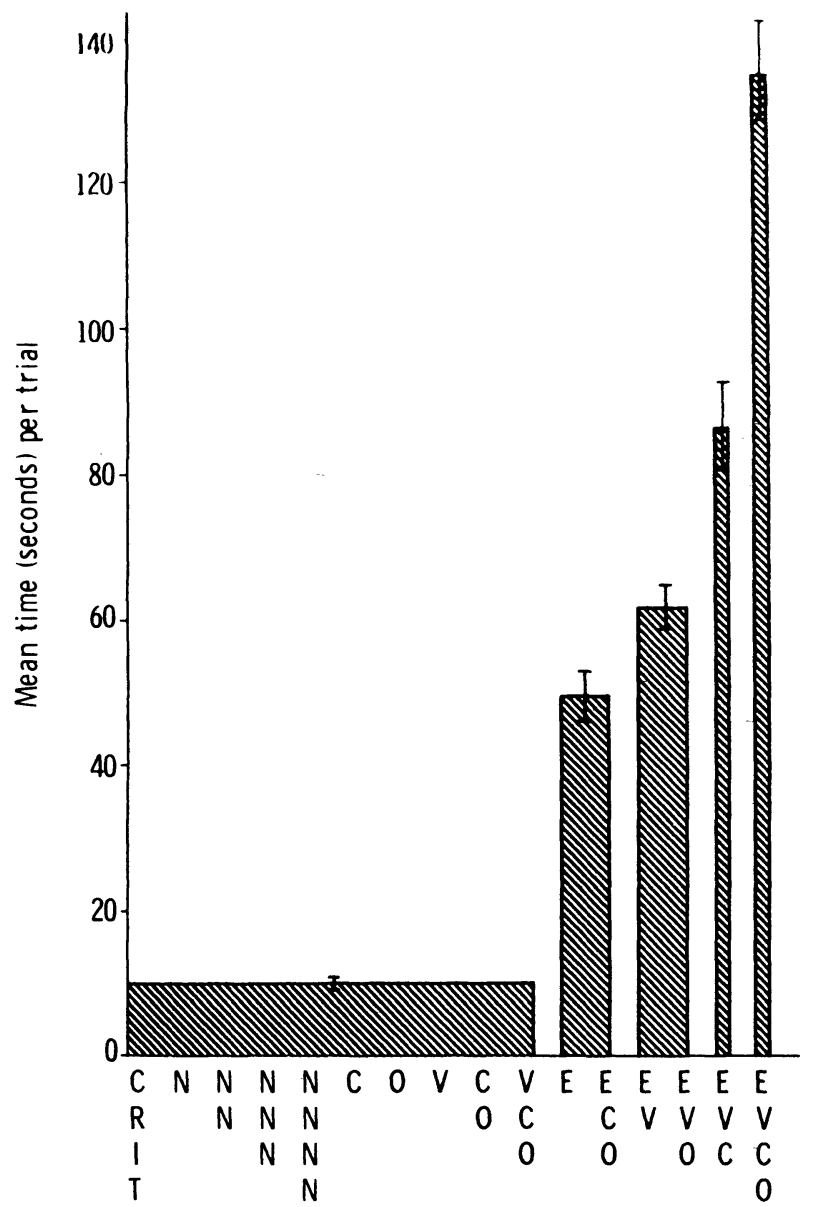

Figure 1. Means and standard errors of seconds/trial for kangaroo rats in various experimental conditions; data are graphed by homogeneous subsets where applicable.

three times that of the $\mathrm{E}$ group, and their collisions about four times that of the E group.

\section{Postmortem Examination}

With three exceptions, all heads and brains were in good condition and showed that the surgeries had been both complete and discrete (Figures 4 and 5).

In Animal No. 1510, the thin walls of the right middle ear cavity were partially crumpled, and the middle ear cavity and cranial cavity contained some hemorrhaged blood. This animal had had no ear surgery. Notes on its training and testing revealed that the damage had probably occurred during training, a few days before criterion was reached, when the animal had struggled and tried to bite while being held by the experimenter. This animal's records reveal no deficit after this time and were consistent with those of other animals in its groups throughout; the damage seems not to have affected its performance.

Animal No. 1542 had slight damage to the tip of the right cerebral cortex, presumably suffered during the removal of the olfactory bulbs which was its first

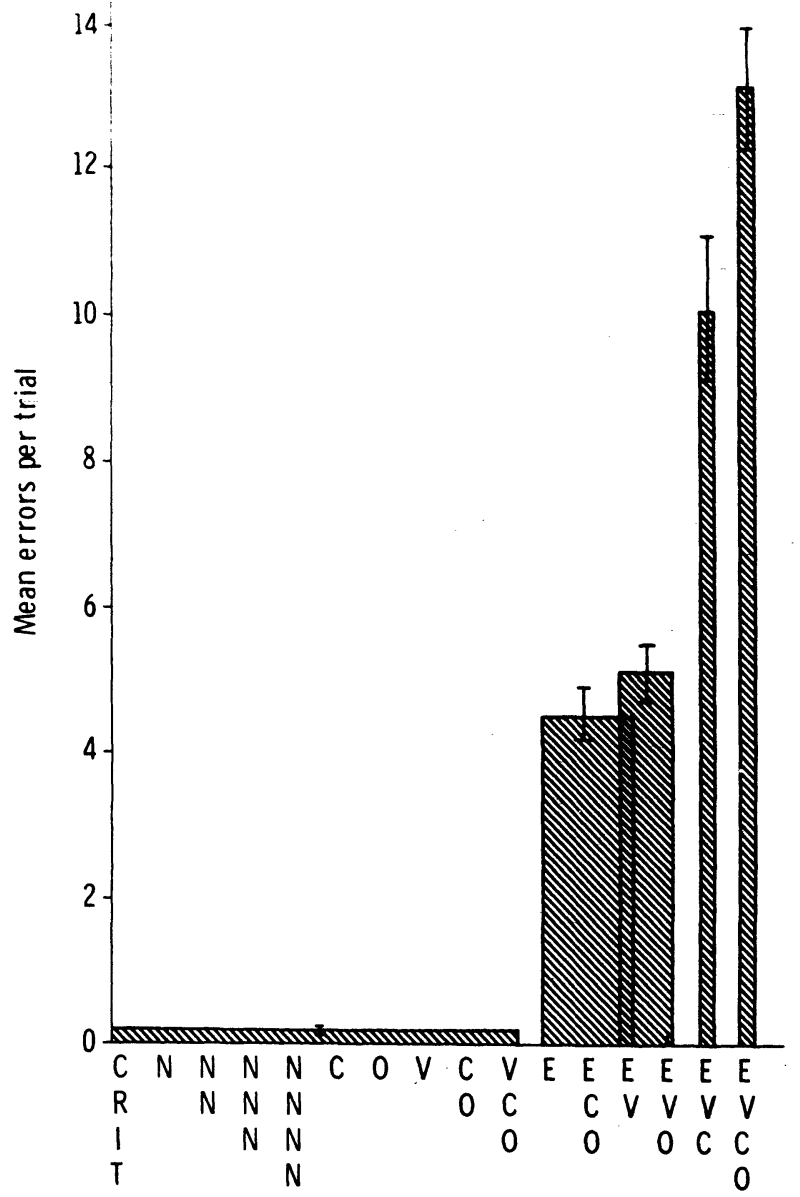

Figure 2. Means and standard errors of errors per trial for kangaroo rats in various experimental conditions; data are graphed by homogeneous subsets where applicable.

surgery. Beginning with the first testing day after this surgery, the animal had had difficulty with its left hind leg and had kept it raised during many trials. Nevertheless, the animal underwent two more deprivations-cochlear destruction and vibrissae removal-running all trials with time, error, and collision records as good as or better than the means of its experimental groups. Then the eyes were removed, and, on the next testing day, the left back leg was found to be paralyzed. No trials were completed that day, and the animal died the following day. (This animal therefore did not contribute to the EVCO data.)

Animal No. 1519 underwent all four surgeries, cochlear destruction being first. On autopsy, it was found to have a severe bilateral middle ear infection with a blood clot in one ear. After three surgeries (ECO), it had made more errors than any other animal in its group (but no collisions), and it was one of two animals in the nine-animal EVCO group that completed none of the 40 trials; with these two exceptions, this animal had performed as well as or better than the means of the animals in its groups. It is possible that the infection had 


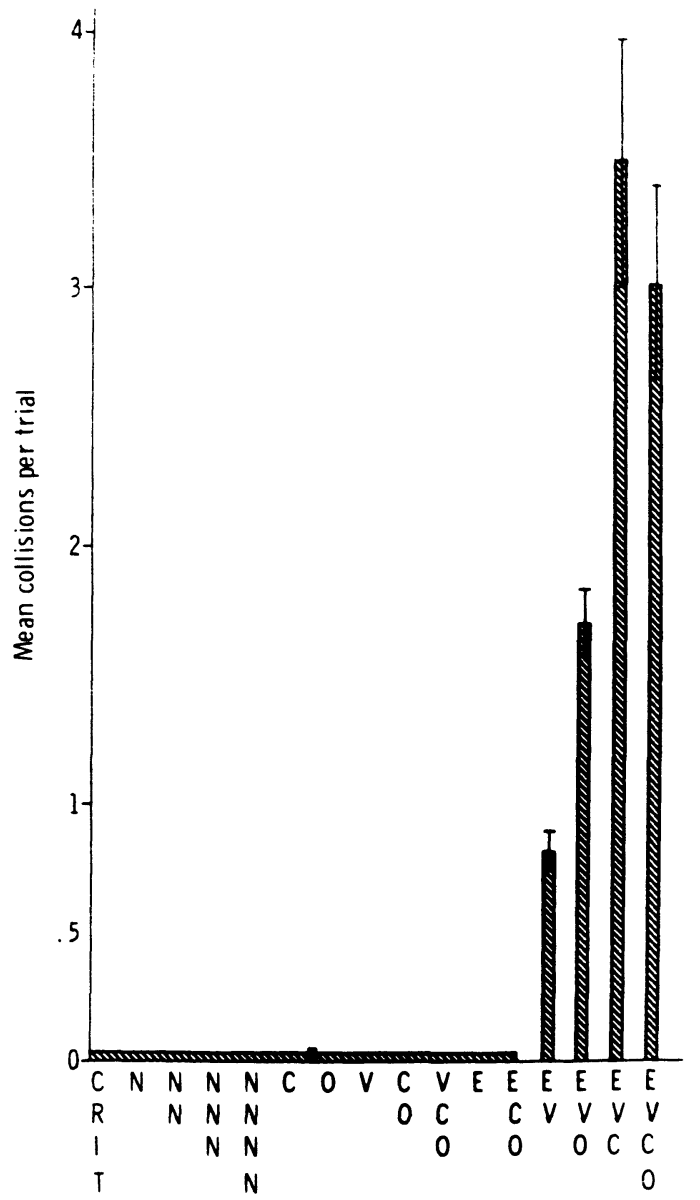

Figure 3. Means and standard errors of collisions per trial for kangaroo rats in various experimental conditions; data are graphed by homogeneous subsets where applicable.

developed to such a point by the fourth postcriterion week that it was responsible for the animal's not running after four surgeries. However, the other EVCO animal that ran no trials showed no abnormalities on autopsy.

\section{DISCUSSION}

Vincent concluded (1912) that the vibrissae were of primary importance in rats' maze learning and performance. Honzik (1936), however, found that vision was of primary importance; he also found that olfaction was necessary when vision was absent or greatly restricted, but that audition was less important and vibrissae even less. Casper (1933) found that no single deprivation caused performance deficits; multiple deprivations showed olfaction and vision to be of equally large importance and audition of little, if any, importance. He concluded that a sense which is normally used may be lost without noticeable effect because another sense is used in its stead.
In contrast to these previous reports, the most striking result of the present study is the dramatic effect of loss of eyes alone and, secondarily, of vibrissae in combination with eyes (but not of vibrissae alone or in combination with other senses). The next most significant result is the strong effect of cochlear destruction, but not of olfactory bulb ablation, when combined with deprivation of both eyes and vibrissae.

While we disagree with Vincent on the primary role of vibrissae, we do agree that loss of vibrissae and eyes together is a serious deprivation.

We were surprised however at the large effect of blinding alone, which seemed inconsistent not only with Casper's results but with our own findings that, for avoiding rattlesnake strikes, kangaroo rats require either hearing or vision (e.g., Webster \& Webster, 1971). In resolving this inconsistency, there are two points to consider in addition to the fact that different tasks are being used in these studies to measure the importance of the senses.

First, it may be that kangaroo rats-which are saltatorial, partially bipedal animals-received more visual information in the alley maze than rats. If so, blinding would be a more serious loss for them, and the serious deficits in performance that followed would be consistent with Honzik's results. They would not be inconsistent with Casper's since, by this theory, we would assume that his animals relied less on vision from the beginning.

Secondly, there may be a large difference in the visual capabilities of the two animals. Vision is said to be poor in the rat (e.g., Barnett, 1963; Vincent, 1912) while it seems well developed in kangaroo rats and other saltatorial rodents (Eisenberg, 1963; Howell, 1932).

Casper's conclusions about the equipotency of vision and olfaction were based only on errors made by deprived animals while relearning the maze. There are some interesting differences, however, in their times to relearn: of those that achieved this criterion, deaf animals took about twice as long, blind about three times as long, and anosmic nearly six times as long as normals.

Our results on olfaction were also surprising. We found that anosmic, deaf anosmic, and vibrissaeless deaf anosmic animals were not unlike normal animals; that blind deaf anosmic animals were not unlike blind animals; and that (except for collisions) blind vibrissaeless anosmic animals were not unlike blind vibrissaeless animals. In contrast to Honzik's and Casper's results with laboratory rats, it seems incontrovertible that olfaction is not an important sense in the maze running of kangaroo rats, although there were plentiful olfactory cues in the maze. This may be because kangaroo rats are solitary in habit; therefore, the scents of other animals may not be particularly relevant to them, and it would seem that their own scents do not 


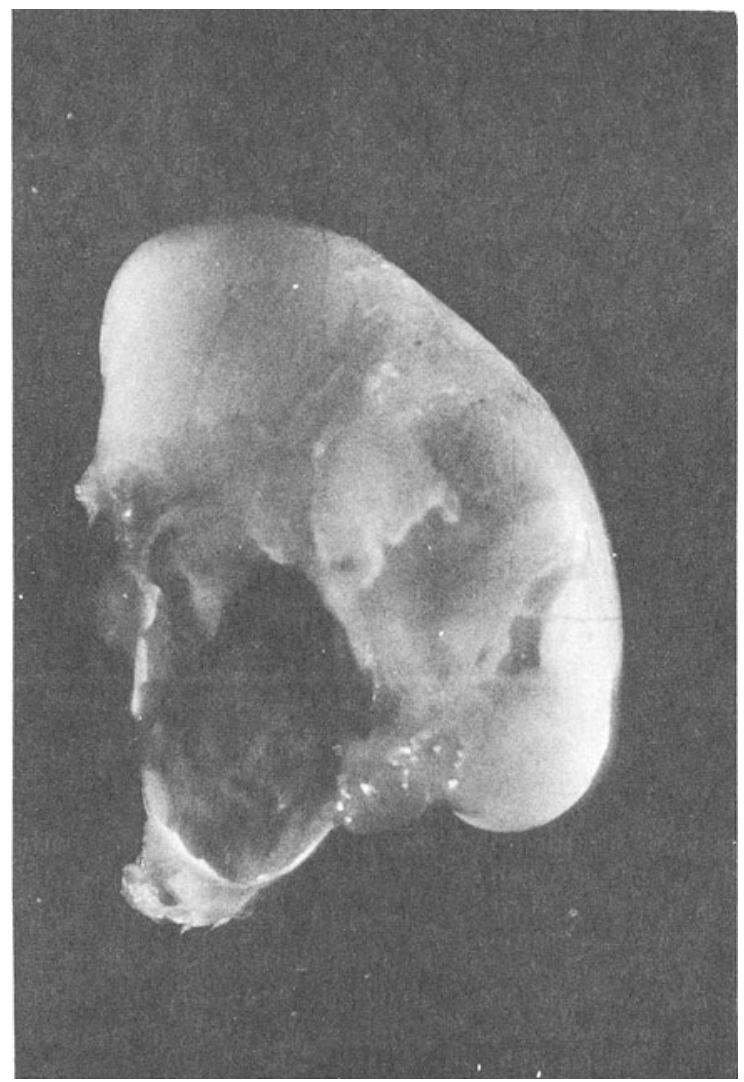

Figure 4. Ventral view of an unperfused brain from a kangaroo rat (No. 1542) whose olfactory bulbs and eyes had been removed, and whose cochleae had been destroyed.

mark trails (or, at least, maze alleys). However, there is evidence that kangaroo rats $(D$. deserti) use olfaction to retrieve seeds buried up to $10 \mathrm{~cm}$ below the surface (Lockard \& Lockard, 1971); and therefore we must consider that while olfaction is not important in their maze running, it is an important sense for kangaroo rats.

On all measures, deprivation of eyes, vibrissae, and cochleae together had a much stronger effect than any other combination of deprivations except EVCO. The consistency and magnitude of this effect led us to suspect some form of echolocation by ultrasonics, such as is found in some other small mammals, even though this would be very surprising in view of the known physical and functional characteristics of these animals' auditory systems. Nevertheless, equipment to record high frequencies was set up in the booth and two animals-an EVC and an EVO-were tested. No ultrasonic or high-frequency vocalizations were recorded.

It is apparent, however, that animals lacking audition, eyes, and vibrissae perform dramatically worse than those lacking eyes alone; or eyes, cochleae, and olfaction; or eyes and vibrissae; or eyes, vibrissae, and olfaction. The ears must be providing the kind of qualitative feedback said to be used by blind persons in

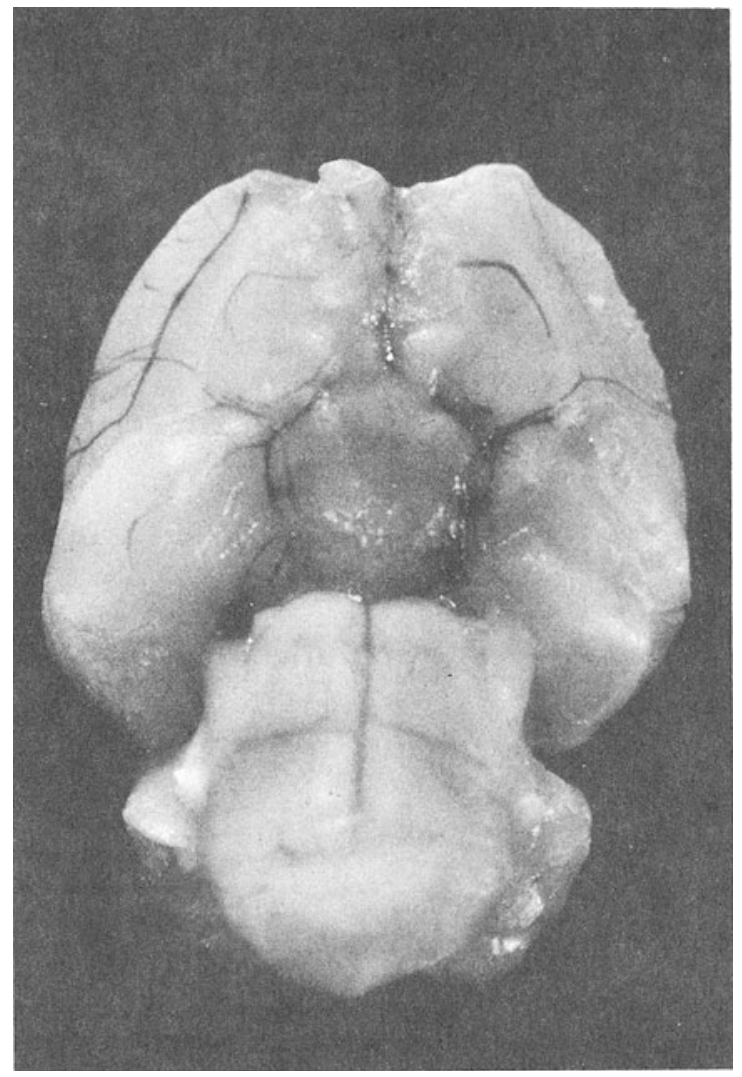

Figure 5. Ventral view of the right temporal bone of the same animal as in Figure 4 after dissection of the ventral wall of the bulla, showing the area where the cochlea had been.

orienting themselves. The fact that kangaroo rats in which audition remains (e.g., EV and EVO) do as poorly as they do may be because their hearing, sensitive in the extreme to low frequencies (e.g., Vernon, Herman, \& Peterson, 1971; Webster \& Webster, 1972), is not adapted to the high frequencies which would provide better directional cues.

An important consideration in the present case is what these data can tell us about the kangaroo rat's sensory world. While maze running does not occur as such in nature, it has some similarity to activities which do. According to Reynolds (1958), Merriam's kangaroo rats spend $75 \%$ of their lives in very simple underground burrows; we found that in one night a $D$. merriami in a fenced-off outdoor plot constructed a burrow resembling a simple $Y$ maze, with arms from 7 to 10 in. long. However, Eisenberg (1963) suggests that the same burrow system may be used by successive generations, being expanded, ramified, and repaired, and thus becoming more complex. Indeed, in some of our field studies, we have found much more complex burrow systems being used by $D$. merriami. These "underground mazes" have several T- and Y-maze turn choices and blind endings. The burrow systems of $D$. spectabilis are even more complex (Vorhies \& Taylor, 1922). 
One would expect that levels of illumination would be very low in such a burrow system and that vision, therefore, would be of little importance in traversing these "mazes"-in contrast to the importance of vision in this study. We must consider, however, which of the measures of this study are relevant to behavior in home burrows. Unless a kangaroo rat is being actively pursued inside its own burrow, its time and errors are probably not relevant: there is no need for great speed, and "errors" are meaningless. It would be important not to collide with the tunnel walls, however; and as we see from the data on collisions, both vibrissae and eyes must be absent before animals make significantly more collisions than normals.

On occasion, snakes enter kangaroo rat burrows, and rapid escape by the kangaroo rat could be necessary. Merriam's kangaroo rats make some tunnels that end blindly close to the surface, as emergency escape routes which can be broken through by a fleeing animal. We have also seen two bannertail kangaroo rats (D. spectabilis), whose burrow was being excavated, dash from the lowest level of the burrow, through the course of the no-longer-existing tunnels and "out" at where an opening had been. Such locomotor behavior appears to be guided not by vision but by kinesthesia.

Maze-running capabilities may also be relevant to the animal's nightly activities aboveground. These include foraging, sand bathing, playing, and some social interaction (Webster \& Webster, Note 1). We have observed, in areas where there is enough vegetation to show it, that, if heteromyids are present, there are also paths worn through the grass or plant cover: and we have occasionally observed kangaroo rats using these paths. We have also seen sand-bathing areas on which many trails converge; apparently, such sites are visited regularly by individuals from several burrows. In short, our observations suggest that kangaroo rats have a regular "beat" which they cover during their nightly activity, and which may take them far from their home burrows (Webster \& Webster, 1971). When danger threatens, the first response is to freeze; and, if necessary, to escape by a series of erratic, last-minute leaps followed by a rapid bipedal retreat to the burrow. If this activity can be likened to maze running, the large effect in these results of loss of vision is interesting and consistent. In an earlier study (Webster \& Webster, 1971), we found that kangaroo rats are more vulnerable to predation during the dark phase of the moon when much of the night is dark or at least only poorly illuminated.

\section{REFERENCE NOTE}

1. Webster \& Webster, unpublished observations.

\section{REFERENCES}

Barnett, S. A. The rat: A study in behaviour. Chicago: Aldine, 1963.

Casper, B. The normal sensory control of the perfected double-alternation spatial-maze habit of the albino rat. Journal of Genetic Psy chology, 1933, 43, 239-292.

Eisenberg, J. F. The behavior of heteromyid rodents. University of California Publications in Zoology, 1963, 69, 1-100.

Honzik, C. H. The sensory basis of maze learning in rats. Comparative Psychology Monographs, 1936, 13, $113 \mathrm{pp}$.

Howell, A. B. The saltatorial rodent Dipodomys: The functional and comparative anatomy of its muscular and osseous systems. Proceedings of the American Academy of Arts and Sciences, 1932, 67, 375-536.

Lockard, R. B., \& Lockard, J. S. Seed preference and buried seed retrieval of Dipodomys deserti. Journal of Mammalogy, 1971, 52, 219-221.

Reynolds, H. G. The ecology of the Merriam Kangaroo rat (Dipodomys merriami Mearns) on the grazing lands of southern Arizona. Ecological Monographs, 1958, 28, 111-127.

Vernon, J., Herman, P., \& Peterson, E. Cochlear potentials in the kangaroo rat, Dipodomys merriami. Physiological Zoology, $1971,44,112-118$.

Vincent, $S$. B. The function of the vibrissae in the behavior of the white rat. Behavior Monographs, 1912, 5. $81 \mathrm{pp}$.

Vorhies, C. T., \& Taylor, W. P. Life history of the kangaroo rat Dipodomys spectabilis spectabilis Merriam. United States Department of Agriculture, Bulletin No. 1091, 1922.

Webster, D. B., \& Webster, M. Adaptive value of hearing and vision in kangaroo rat predator avoidance. Brain, Behavior and Evolution, $1971,4,310-322$.

Webster, D. B., \& Webster, M. Kangaroo rat auditory thresholds before and after middle ear reduction. Brain, Behavior and Evolution, 1972, 5, 41-53.

Winer, B. J. Statistical principles in experimental design. New York: McGraw-Hill, 1962.

(Received for publication October 31, 1974 revision accepted January 30,1975 .) 